\title{
Nachruf auf Professor Sooyoung Oh
}

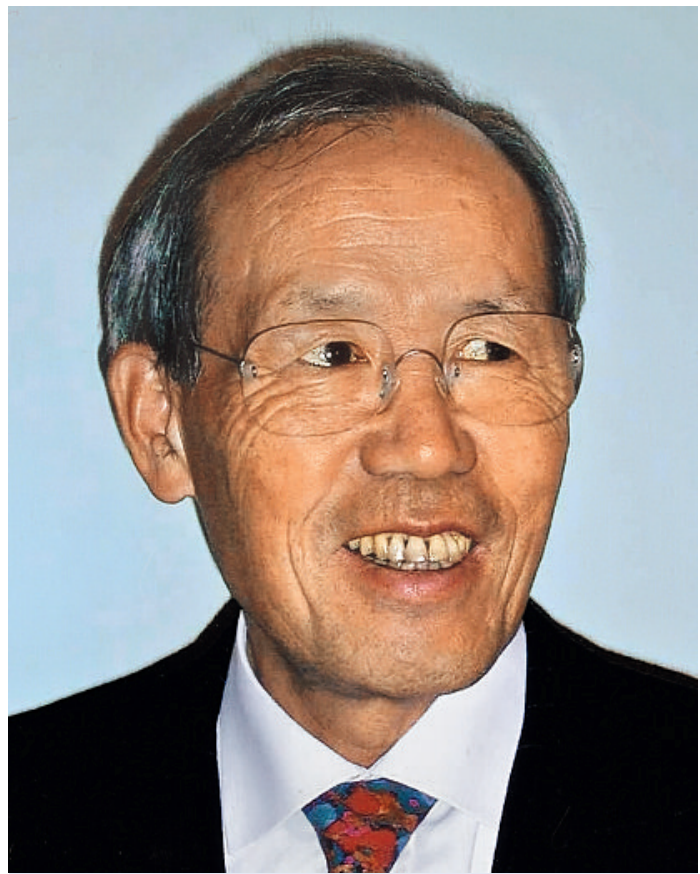

Am 15. August 2010 ist nach kurzer, schwerer Krankheit Professor Sooyoung Oh in Chur verstorben. Er war in mehrfacher Hinsicht eine besondere Persönlichkeit unter den schweizerischen Neurochirurgen.

\section{Jugend und beruflicher Werdegang \\ in Südkorea und in der Schweiz}

Sooyoung Oh wurde am 3.11.1932 in Yoju, östlich von Seoul, geboren. Korea war damals noch von Japan besetzt. Die Familie Oh führte eine Sägerei. Der begabte Sooyoung, der jüngste von vier Kindern, wurde von einem Onkel, der Professor für Pharmakologie an der Staatlichen Universität von Seoul war, gefördert. Er studierte in Seoul zunächst Zahnmedizin und dann Humanmedizin, die er 1960 abschloss. Auf $2 \frac{1}{2}$ Jahre Militärdienst als Sanitätsleutnant folgte während dreier Jahre eine Weiterbildung an der Postgraduate School of Medicine mit dem Diplom als Master of Medical Science. In seiner ersten Ehe mit Young-In Woo ab 1963 wurden zwei Kinder geboren.

Sooyoung Oh's Wunsch war es, Neurochirurg zu werden. Dies veranlasste ihn 1966, in die Schweiz zu kommen. Während $1 \frac{1}{2}$ Jahren bildete er sich in Locarno in Allgemeiner Chirurgie aus und Mitte 1967 begann er seine Weiterbildung in Neurochirurgie bei Professor Hans Markwalder an der Neurochirurgischen Universitätsklinik in Bern. Hier erlebte ich ihn auch während eines Jahres als Assistent an der Neurologischen Klinik und dann bei den regelmässigen
Kontakten mit der Neurochirurgie. Er war von grösster Hilfsbereitschaft, von grenzenloser Einsatzbereitschaft und stets verfügbar. Er war sehr rasch in der Schweiz integriert, absolvierte auch ein Praktikum bei Prof. Feremutsch in der Anatomie in Bern und wurde 1971 Oberarzt an der Neurochirurgie.

Er verfasste eine Dissertation (RickhamklappenDrainage bei rezidivierendem zystischem Kraniopharyngeom, Schweiz.Arch.Neurol.Neurochir. Psychiatrie 113, 57-72, 1973), so dass er 1973 den schweizerischen Facharzttitel für Neurochirurgie erwarb. Die erste Ehe ging auseinander, und Sooyoung Oh heiratete die Schweizerin Christine Graf. Sein drittes Kind, die Tochter Jacqueline, wurde 1977 geboren.

*

\section{Neurochirurgie in Chur, Goldach und Niederuzwil}

1972 wurde Dr. Oh als Leiter der von seinem Vorgänger Prof. Charles Probst begründeten ersten in der Schweiz eröffneten, nicht universitären Neurochirurgischen Abteilung am Kantonsspital Graubünden berufen. Dieses Amt übte er bis 1989, also während rund 17 Jahren, aus. Daneben wirkte er von 1974 bis 1983 auch noch als Lehrbeauftragter an der Neurochirurgischen Universitätsklinik Bern. Die Tätigkeit in Chur war gekennzeichnet durch die Knappheit an Mitteln, die vom verantwortlichen Leiter einen schon damals aussergewöhnlichen, aus heutiger Sicht physisch nicht mehr zumutbaren persönlichen Einsatz rund um die Uhr, und dies während praktisch 365 Tagen pro Jahr, forderte.

Ihm standen nämlich während der ersten Jahre seiner Amtszeit lediglich ein eigener Assistent und ein rotierender Assistent der Chirurgie zur Verfügung. Erst 1976 wurde ihm ein Oberarzt bewilligt. Somit musste er mehr oder weniger ständig selber präsent und verfügbar sein. Die damals noch schlecht gesicherte, aber intensiv befahrene Alpentransversale via San Bernardino, die Berge und der Wintersport forderten ihren regelmässigen Tribut. Schädel-HirnVerletzungen, oft im Rahmen schwerer Polytraumen, prägten deshalb seinen Alltag im Notfalldienst, und dies in touristischen Spitzenzeiten rund um die Uhr. Die damals von oft schweren Hirnverletzungen betroffenen Patienten, die direkt ab Skipiste per Helikopter ins Kantonsspital geflogen worden waren, veranlassten ihn, über die vielfältigen Ursachen von Kopfverletzungen nach Pistenunfällen ein zum Teil von ihm selbst mit Unfall-Zeichnungen illustriertes und mit mechanischen Experimenten angereichertes 
Buch zu publizieren («Prevention of Head Injuries in Skiing», Karger 1985).

Er propagierte wohl als erster in der Schweiz das Tragen von Schutzhelmen besonders für Kinder beim Skifahren - und trug auch selber immer einen solchen. Die finanziellen Mittel des Kantons waren damals beschränkt, viele technische Neuerungen erreichten das Spital nur mit Verzögerung. Als zum Beispiel die Computertomographie andernorts schon zur unverzichtbaren Diagnostik von chirurgisch behandelbaren Rücken- und Hirnerkrankungen gehörte, beschränkte sich die neuroradiologische Diagnostik in den ersten Jahren von Dr. Oh's Tätigkeit im Kantonsspital Graubünden noch auf die Karotisangiographie, die Pneumoenzephalographie, die Myelographie zum Nachweis von Raumforderungen im Hirn und in der Wirbelsäule.

So erinnern sich seine ehemaligen Schüler, die ihre Chirurgie heute mit einer Palette von modernsten neuroradiologischen Techniken planen, mit Verwunderung an die damaligen Verhältnisse in den 70er und frühen 80er Jahren, als die Patienten gegebenenfalls zur CT-Untersuchung mit dem Helikopter von Chur nach Zürich geflogen oder der Assistent mit schwer interpretierbaren Bildern an die Uni Bern geschickt wurde.

Prof. Oh war ein engagierter, manuell sehr geschickter Chirurg mit einem breiten operativen Spektrum. Er war im besonderen ein versierter Chirurg der Halswirbelsäule mit dem vorderen Zugang nach Cloward und hatte viel Erfahrung mit dem posterolateralen Zugang zur Lendenwirbelsäule und der interkorporellen Spondylodese, deren Technik er erweiterte. 1986 ernannte ihn die Universität von Seoul in seinem Heimatland Korea zum Clinical Professor. Als sein ehemaliger neurologischer Lehrer erinnere ich mich, wie er mich anlässlich eines Vortrages in Seoul begleitete und mit welchem Respekt die koreanischen Kollegen ihm begegneten.

Spannungen mit der Chirurgischen Leitung in Chur veranlassten 1989 seinen Rücktritt. Seither führte er einerseits eine Privatpraxis als Neurochirurg in Chur, vor allem aber war er fortan in der Klinik St. Georg in Goldach SG und in der Klinik Marienfried in Niederuzwil SG als Neurochirurg tätig. Wissenschaftlich hat er neben dem soeben erwähnten Buch über Schädel-
hirn-Traumata an einem Buch mit dem Titel «Freizeitunfälle im Kindes- und Jugendalter» als Co-Autor gewirkt (Schütze Udo Hrsg. Thieme 1992). Im Jahr 2009 erschien bei Karger seine Monographie über «Autologous Bone Plugs Fusion. Treatment for Lumbar Instability». In dieser Monographie verwendet er, seine Erfahrungen an der Halswirbelsäule auf die lumbale Wirbelsäule übertragend, eine weitgehend auf Fremdmaterial verzichtende Technik der Versteifung der Lendenwirbelsäule.

\section{Der Mensch «dahinter» und «danach»}

In den letzten Jahren zog er sich von der ärztlichen Tätigkeit zurück. Er spielte mit Leidenschaft Golf, präsidierte von 2004-2007 den Korean-Swiss-SeniorClub und vor allem zeichnete er und malte, worin er sehr begabt war. Die öffentlich zugänglichen Räume der Klinik St.Georg waren beispielsweise reich mit seinen in koreanischem Stil gemalten Werken dekoriert. Seine ehemaligen Assistenten bestätigen einerseits sein aussergewöhnliches manuelles Geschick als Operateur und andererseits seinen beispielhaft unermüdlichen und Leben rettenden Einsatz für seine Patienten. Vielen seiner neurochirurgischen Mitarbeiter war er ein hilfsbereiter und geduldiger Lehrer und Seniorassistent im Operationssaal und ein wohlwollender, humorvoller Vorgesetzter.

Professor Oh stammte aus dem fernen und uns in seiner Mentalität unvertrauten Korea. Seit 1966 lebte er in der Schweiz, hat sich in unserem Land perfekt integriert und wurde im November 1985 Schweizer Bürger. Er hat während 17 Jahren die Neurochirurgische Abteilung eines grossen Kantonsspitals geleitet. Er ist ein klassisches Beispiel dafür, wie die Medizin, namentlich im respektvollen Umgang mit den jungen Mitarbeitern und im selbstlosen Einsatz für die Patienten, keine nationalen Grenzen kennt und wie unabhängig von der Herkunft Ärzte überall die gleichen Ideale und Ziele leben können.

Prof. Dr. med. Marco Mumenthaler, Zürich Prof. Dr. med. Urs D. Schmid, Zürich Dr. med. Matthias Rohner, Bern 